\title{
Exposición a la violencia, violencia filioparental y en el noviazgo de jóvenes mexicanos
}

\author{
Exposure to parental violence, child to parent violence and dating violence of Mexican youth
}

\author{
Daniela Cancino-Padilla ${ }^{1}$, Christian Alexis Romero-Méndez ${ }^{2}$ y José Luis Rojas-Solís ${ }^{3 *}$ \\ ${ }^{1}$ Universidad Juárez Autónoma de Tabasco, México. \\ ${ }^{2}$ Universidad del Valle de Puebla, México. \\ ${ }^{3}$ Benemérita Universidad Autónoma de Puebla, México.
}

* Correspondencia: José Luis Rojas-Solís. Facultad de Psicología, Benemérita Universidad Autónoma de Puebla. Calle 4 Sur \#403, Centro Histórico, 72000. Puebla, Puebla (México). Correo electrónico: ilrojassolis@gmail.com

Recibido: 19 de febrero de 2020 | Revisado: 07 de marzo de 2020 | Aceptado: 26 de marzo de 2020 | Publicado Online: 01 de abril de 2020.

CITAR COMO-

Cancino-Padilla, D., Romero-Méndez, C., \& Rojas-Solís, J. (2020). Exposición a la violencia, violencia filioparental y en el noviazgo de jóvenes mexicanos. 1 Interacciones, 6(2), e228. http://dx.doi.org/10.24016/2020.v6n2.228

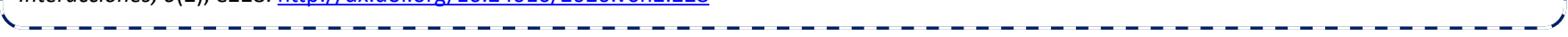

\section{RESUMEN}

Introducción: La violencia es un problema grave que ha generado preocupación a nivel mundial a causa de las consecuencias que genera en quienes la sufren. Sin embargo, aunque ha sido estudiada en sus diversas formas, el estudio de la violencia filioparental aún conserva considerables lagunas en su investigación. Por lo tanto, este trabajo tuvo el objetivo de analizar la violencia filioparental, hacia la pareja y la observada entre los padres para identificar su frecuencia, así como también las posibles correlaciones entre ellas. Método: La muestra final se integró de 256 individuos de entre 18 y 30 años. Se emplearon el cuestionario de violencia filioparental, la escala de táctica de conflictos, en su versión modificada. Resultados: Los resultados indicaron diferencias entre sexos respecto a la incidencia tanto de la violencia filioparental como hacia la pareja, al igual que correlaciones entre las variables estudiadas y la bidireccionalidad en la violencia. Conclusiones: Resulta importante indagar más en dichos fenómenos para comprenderlos mejor, tomar las medidas necesarias y mejorar los programas de prevención e intervención.

Palabras clave: Violencia filioparental; Violencia en el noviazgo; Violencia interparental; México.

\begin{abstract}
Background: Violence is a serious problem that has generated worldwide concern because of the consequences it generates on those who suffer it. However, although it has been studied in its various forms, the study of violence against parents still has considerable research gaps. Therefore, this work had the objective of analyzing violence against parents, dating violence and observed between the parents to identify their frequency, as well as the possible correlations between them. Methods: The final sample was made up of 256 individuals between 18 and 30 years old. The Child to Parent Aggression Questionnaire-Revised and the Conflict Tactics Scale - Modified version were used. Results: The results indicated differences between the sexes regarding the incidence of both violence against parents and dating violence, as well as correlations between the variables studied and the bidirectionality in violence. Conclusion: It is important to investigate these phenomena more to understand them better, take the necessary measures and improve prevention and intervention programs.
\end{abstract}

Key words: Child to Parent Violence; Dating Violence; Interparental Violence; Mexico. 


\section{INTRODUCCIÓN}

La violencia que surge en las relaciones de pareja, como el noviazgo, es un problema que ha generado preocupación a nivel mundial; se trata de un fenómeno silencioso que afecta de forma grave a quien la padece, añadido a ello parece que los grupos vulnerables que la sufren son cada vez más; por ello resulta importante su entendimiento para así poder mejorar las acciones de prevención y así reducir sus devastadores efectos en la sociedad (Batiza, 2017; Bayona, Chivita y Gaitan, 2015; Dardis, Dixon, Edwards y Turchik, 2015). Al menos en México la violencia psicológica es el tipo de violencia en la pareja con mayor prevalencia en las mujeres de 15 años y más (40.1\%), seguida por la violencia económica $(20.9 \%)$, la violencia física $(17.9 \%)$ y por último la violencia sexual (6.5\%) (Instituto Nacional de Estadística Geografía e Informática, 2016).

En ese sentido, la violencia en el noviazgo se puede definir como la amenaza o el uso de la fuerza física, restricción, abuso psicológico y/o abuso sexual, que causan daño o malestar a la pareja (Morales y Rodríguez, 2012). Se trata de un fenómeno multifacéticos, pues se puede presentar en distintas formas así, de acuerdo con Leen et al. (2013), la violencia en el noviazgo se puede clasificar en tres grandes tipos: 1) Violencia física, que hace referencias al uso intencional de la fuerza física con el potencial de causar muerte, discapacidad, lesión o daño. 2) Violencia psicológica, que involucra trauma causado por actos, amenazas o tácticas coercitivas, como humillar, controlar, retener información o hacer algo para que la víctima se sienta disminuida o avergonzada. Y 3) Violencia sexual, que incluye tres elementos: a) el uso de la fuerza física para obligar a una persona a participar en un acto sexual en contra de su voluntad, b) involucra a un individuo en un acto sexual intentado o completado, quien no puede comprender la naturaleza o rechazar la participación en el mismo, y c) contacto sexual intencional, no deseado o tocamiento intencional a alguien con capacidad disminuida. Sin detrimento de lo anterior, es preciso señalar que en años recientes se ha incrementado poco a poco el reconocimiento y estudio de una "nueva" forma de violencia en la pareja, aquella ejercida a través de medios electrónicos entre las que destaca el ciberacoso y la ciberviolencia de pareja (Caridade, Braga y Borrajo, 2019; Romo-Tobón, Vázquez-Sánchez, Rojas-Solís y Alvídrez, 2020).

En la literatura científica internacional, la prevalencia de los diversos tipos de violencia en el noviazgo muestra una gran variabilidad, pese a ello podría decirse que la violencia psicológica es la más reportada por los jóvenes, en ese sentido se sugiere también una mayor prevalencia en la perpetración de la violencia psicológica por parte de las mujeres y sexual por los hombres (Alegría y Rodríguez, 2015; Rey-Anacona, 2013); al respecto cabe mencionar que en muchos estudios la naturaleza de la violencia es bidireccional, es decir ambos sexos pueden ser perpetradores y víctimas de violencia (Rojas-Solís, 2013; Rubio-Garay et al., 2017). Ahora bien, se han elaborado numerosas teorías para la explicación del fenómeno, entre las cuales destacan la del aprendizaje social y la sistémica. De acuerdo con la primera, el mecanismo principal de aprendizaje de las conductas violentas es el aprendizaje observacional en el entorno familiar, donde se pueden desarrollar una observación y vivencia de la violencia, al igual que una pronunciada identificación en el observador a causa de su relación afectiva con el modelo (Bandura, 1982). Por otra parte, desde la perspectiva sistémica se señala la existencia de múltiples variables estrechamente relacionadas con la dinámica de la pareja e involucradas en el origen y mantenimiento de las conductas violentas como son los patrones de relación, comunicación, respuestas o resolución de conflictos; aunado a ello es indispensable considerar que el fenómeno involucra a todos sus miembros, pues la violencia en la pareja no puede ser atribuida a solo unos de ellos (Ospina y Clavijo, 2016).

Así, entre las variables más relacionadas con la violencia en el noviazgo se encuentran: las actitudes de justificación de la violencia, la influencia de los iguales, la exposición a la violencia dentro de la propia familia o en la comunidad, una historia de maltrato físico y psicológico, de abuso sexual y de hábitos de crianza negativos, los estereotipos tradicionales de género, un déficit de habilidades sociales y de comunicación, un manejo inadecuado de la ira, una baja autoestima, el consumo de alcohol y otras drogas, una historia personal de agresión, la falta de empatía y la carencia de apoyo social (Rubio-Garay et al., 2015).

Siguiendo el mismo orden de ideas, la exposición a la violencia dentro de la familia, y de manera particular la observada entre los padres, es un factor de riesgo para la violencia en el noviazgo, pues diversos estudios han investigado la relación existente entre dichas variables, encontrando que los miembros provenientes de familias disfuncionales manifestaron mayor incidencia de violencia en el noviazgo, y asociaciones significativas entre haber presenciado conductas violentas entre los progenitores y la perpetración de violencia contra la pareja (Alvarado, 2015; Bolívar, Rey y Martínez, 2017; Makin-Byrd y Bierman, 2013; Martínez, Vargas y Novoa, 2016).

Por otro lado, algunas de las consecuencias de la violencia en el noviazgo pueden ser el bajo rendimiento académico, deserción escolar, insatisfacción con la relación, baja autoestima, inseguridad, aislamiento, trastornos alimentarios, ansiosos y depresivos, síntomas de estrés postraumático, ideación suicida, normalización de la violencia y por lo tanto, riesgo de ser victimizadas en las relaciones de pareja adultas futuras, disminución del uso de métodos anticonceptivos y embarazo precoz (Valdivia y González, 2014).

\section{Violencia filioparental}

Considerando que la violencia interpersonal es un fenómeno multifactorial y multifacético, últimamente la atención se ha enfocado en una manifestación antes ignorada, se trata de la violencia de los hijos contra sus padres o figuras de autoridad, la cual es conocida como violencia filioparental (VFP; Rojas-Solís, Vázquez-Aramburu y Llamazares-Rojo, 2016). Si bien es cierto que la violencia de hijos hacia sus progenitores no es un fenómeno realmente nuevo, en países como México los estudios sobre esta problemática 
aún siguen siendo escasos por lo que resulta necesario brindar mayor atención y avivar el interés sobre tal problemática en la comunidad científica (Molla-Esparza y Aroca-Montolío, 2018; Vázquez-Sánchez et al., 2019).

Sin detrimento de lo anterior, podría entenderse la VFP, según Llamazares, Vázquez y Zuñeda (2013) y Pereira et al. (2017), como todo acto perjudicial reiterado ya sea físico, psicológico o económico que los hijos realizan contra las y los progenitores o cualquier otra figura (familiar o no) que ocupe su lugar, con el objetivo principal y último de ganar poder y/o control sobre estos, alcanzando también en este proceso diferentes objetivos específicos (materiales u otro tipo de beneficios). Es así como los o las hijas pueden manifestar tres tipos de conductas: 1) Violencia física, que comprende acciones que pueden producir daño corporal y heridas; 2) Violencia psicológica, la cual engloba comportamientos que atentan contra los sentimientos y las necesidades afectivas de un individuo; y 3) Violencia económica, que implica conductas que restringen las posibilidades de ingresos y/o ahorros de los progenitores a través de robos, venta o destrucción de objetos y generación de deudas (Aroca-Montolío, Lorenzo-Moledo y Miró-Pérez, 2014).

Con respecto a la prevalencia de la VFP, la mayoría de los estudios señalan que los adolescentes varones son quienes ejercen mayormente la violencia hacia sus padres (con un porcentaje del $60 \%$ al $80 \%$ del total), mientras que la mayor parte de las investigaciones afirman que las figuras femeninas (madres $u$ otras cuidadoras como las abuelas) son por lo habitual el centro del maltrato (Martínez et al., 2015). Ahora bien, algunos posibles predictores de la VFP son el consumo abusivo de sustancias, como las drogas y el alcohol y, por otra parte, la presencia de factores previos, como la dificultad de los padres para cumplir normas y respetar limites, o la influencia de las amistades (negativas) y otros modelos familiares (del Moral et al., 2015). En consonancia con lo anterior también se encuentran modelos que sugieren la observación como principal mecanismo de aprendizaje de conductas violentas en el ámbito familiar, tener amigos que manifiesten la VFP, la aprobación de conductas delincuenciales, el percibir poco o nulo control externo y, por último, presentar lazos de unión débiles con los padres (Rojas-Solís et al., 2016).

Considerando que la violencia es un fenómeno interpersonal que se suele estudiar de manera fragmentada, inconexa o separada, aunque "se ha encontrado que estos diferentes tipos de violencia (en sus distintas modalidades) no suelen presentarse por separado, coexistiendo, lo que se conoce como co-ocurrencia de violencia, es decir la presencia simultánea de diferentes formas de violencia" (Hamby \& Grych, 2013 citado por Espinobarros-Nava, Muñoz-Ponce y Rojas-Solís, 2018, p. 156) , la presente investigación tiene el objetivo de explorar si hay presencia de violencia en el noviazgo y su co-ocurrencia con la VFP y la observada entre los padres, con el fin de determinar posibles correlaciones entre dichos fenómenos.

En ese sentido, los objetivos específicos son analizar la frecuencia de violencia en las relaciones de pareja, de violencia observada en los padres y de VFP, identificar si hay diferencias por sexo, y por último, encontrar las asociaciones que hay entre la violencia en las relaciones de pareja, la violencia observada en los padres y la VFP. Las hipótesis que se derivan de los objetivos mencionados anteriormente se exponen a continuación:

1. La violencia filioparental se cometerá con mayor frecuencia contra la madre.

2. Las mujeres ejercerán con mayor frecuencia la violencia filioparental psicológica, y los hombres la violencia filioparental física.

3. En sus relaciones de pareja las mujeres ejercerán con mayor frecuencia la violencia psicológica, y los hombres la violencia física.

4. El haber sufrido algún tipo de violencia en la pareja se asociará con la perpetración de la misma, es decir la violencia de pareja se presentará de manera bidireccional.

\section{MÉTODO}

Diseño

Estudio con enfoque cuantitativo, diseño no experimental, transversal y ex post facto, con fines exploratorios, descriptivos y correlacionales.

\section{Participantes}

La muestra se integró por estudiantes universitarios, la mayoría procedentes del estado de Tabasco (México), para lo cual se establecieron los siguientes criterios de inclusión: tener entre 18 y 30 años de edad, tener o haber tenido al menos una relación de pareja; y haber vivido o vivir con ambos padres. Al principio se obtuvieron las respuestas de 338 individuos, pero siguiendo los criterios de inclusión, se descartaron las respuestas de aquellos que no habían vivido o no vivían con ambos padres. La muestra final se conformó de 256 sujetos ( $68 \%$ mujeres y $32 \%$ hombres), con edades comprendidas entre los 18 y 30 años $(M=21, D T=1.84)$ todos con orientación heterosexual. La media de duración de la relación actual en meses fue $M=26.06(D T=20.64)$ y de la relación pasada $M=11.48$ (DT=15.06).

\section{Instrumentos}

Al inicio se realizó un estudio sociodemográfico para recolectar datos generales, seguido de la implementación de los siguientes instrumentos:

The Adolescent Child-to-Parent Aggression Questionnaire (Calvete et al., 2013), que evalúa la violencia cometida por los individuos hacia sus padres y se compone de 10 reactivos para evaluar la violencia hacia la madre, y 10 reactivos para evaluar la misma hacia el padre. El instrumento se integra de dos subescalas: la de VFP física y la de VFP psicológica. Los ítems se evalúan con la escala de respuesta Likert de 4 anclajes ( $0=$ Nunca-esto no ha pasado en mi relación con mi madre o padre, 1=Rara vez-únicamente ha sucedido en 1 ó 2 ocasiones, 2=Algunas veces-ha ocurrido entre 3 y 5 veces y $3=$ Muy a menudo-se ha dado en 6 o más ocasiones). 
La validación del cuestionario en mexicanos presentó buenas propiedades psicométricas, su confiabilidad se obtuvo por medio del coeficiente de alfa, obteniendo índices altos (Calvete y Veytia, 2018). Ahora bien, los índices de consistencia interna ( $\alpha$ ) obtenidos en la presente investigación para esta escala y los demás instrumentos pueden observarse a detalle en la Tabla 1.

The Conflicts Tactics Scale (Straus, 1979), se utilizó para identificar las conductas violentas observadas entre los padres. La escala mide la forma en la que los progenitores resuelven sus conflictos como pareja e incluye tres subescalas: violencia psicológica, violencia física leve y violencia física grave. Los ítems se evalúan con la escala de respuesta Likert de 5 anclajes (1=Nunca, 2=Rara vez, 3=Algunas veces, 4=A Menudo y 5=Muy a menudo). Esta escala ha sido validada para población mexicana y demostrado buenos índices de consistencia interna (Straus y Mickey, 2012).

The Modified Conflicts Tactic Scale (Muñoz-Rivas et al., 2007): se incluyó para reconocer la presencia de la violencia en la relación de pareja. La escala evalúa la manera de los individuos de resolver conflictos con sus parejas e incluye ítems de doble naturaleza, mostrando información tanto de la violencia cometida como de la sufrida. El instrumento se divide en tres subescalas: violencia psicológica, violencia física leve y violencia física grave. Los reactivos se evalúan con la escala de respuesta Likert de 5 anclajes (1=Nunca, 2=Rara vez, 3=Algunas veces, 4=A Menudo y 5=Muy a menudo). La adaptación y validación en población mexicana fue realizada por Ronzón-Tirado, Muñoz-Rivas, Zamarrón y Redondo (2019) quienes obtuvieron índices altos de confiabilidad.

\section{Procedimiento}

Una vez conformado el instrumento virtual de evaluación, se procedió a difundir el enlace para responderlo a través de la plataforma de Google Forms, de manera que los participantes podían responder desde sus dispositivos móviles u ordenadores en el horario que les resultará más conveniente y cómodo, con una duración de aproximadamente 20 minutos. Al momento de compartir el enlace del cuestionario se realizó una presentación general de los objetivos de la investigación, e igualmente se hizo énfasis en la confidencialidad de las respuestas asegurando el anonimato.

\section{Aspectos éticos}

En cuanto a los aspectos éticos, es preciso hacer hincapié en que se adoptaron las medidas sugeridas por la Sociedad Mexicana de Psicología (2007) así como aquellas propias de la investigación psicológica desarrollada a través de medios virtuales (Eynon, Schroeder y Fry, 2012; Nosek, Banaji y Greenwald, 2002).

\section{Análisis de datos}

Los análisis se realizaron a través del programa SPSS v. 22 para Windows, iniciando con los análisis descriptivos e inferenciales y con los análisis de fiabilidad de las subescalas utilizadas por medio del alfa de Cronbach. Para verificar la consistencia interna los valores deben ser superiores a 0.7 (Nunnaly y Bernstein (1994). Posteriormente se procedió con las pruebas de normalidad, y tras detectar no normalidad en las respuestas, se llevaron a cabo análisis no paramétricos, como la prueba $U$ de Mann Whitney para detectar diferencias significativas entre mujeres y hombres. Para determinar el tamaño del efecto se utilizaron los criterios de Cohen (1988) para la $r$ de Rosenthal $\left(r_{\text {bis }}\right): 0.1=$ efecto pequeño, $0.3=$ efecto mediano, $0.5=$ efecto grande. Para finalizar, con el objetivo de analizar las asociaciones existentes entre las variables se utilizó el coeficiente de correlación de Spearman $\left(r_{h o}\right)$.

\section{RESULTADOS}

\section{Consistencia interna}

Para empezar, se realizó el análisis de fiabilidad de las subescalas utilizadas por medio del alfa de Cronbach, obteniéndose niveles muy aceptables, siguiendo los criterios sugeridos por Nunnaly y Bernstein (1994), tanto en mujeres como en hombres, especialmente en las subescalas de violencia física leve cometida por el padre (.91 en mujeres y .92 en hombres) y de violencia física leve sufrida (.89 en mujeres y .95 en hombres) (Ver Tabla 1 ).

\section{Prevalencia de violencia filioparental}

Como se puede observar en la Tabla 2, en la muestra total (mujeres y hombres) es mayor la incidencia de VFP psicológica hacia la madre, ya que 215 participantes señalaron que al menos una vez cometieron este tipo de actos. Y respecto a la VFP física, es mayor su incidencia hacia el padre, con 28 participantes que indicaron haberla ejercido al menos una vez.

\section{Diferencias por sexo en las variables estudiadas}

Los resultados de la prueba $U$ de Mann-Whitney, revelaron diferencias significativas en varios tipos de violencia. (Ver Tabla 3), aunque de acuerdo con los criterios de Cohen (1988) para determinar el tamaño del efecto (.1=efecto pequeño, .3=efecto mediano, .5=efecto grande), éste fue pequeño en la mayoría de los casos. Por ejemplo, las jóvenes manifestaron haber perpetrado con mayor frecuencia violencia psicológica hacia sus progenitoras, que los varones $\left(U=5806, Z=-2.41, p=0.01, r_{\text {bis }}=-0.15\right)$.

Asimismo, en el caso de la violencia psicológica cometida por la madre $\left(U=5777.5, Z=-2.46, p=0.01, r_{\text {bis }}=-0.15\right)$ y la violencia física leve cometida $\left(U=5893.5, Z=-2.28, p=0.02, r_{\text {bis }}=-0.14\right)$ por la madre, las mujeres señalaron haber observado este fenómeno más que los hombres. Las participantes quienes indicaron haber cometido violencia psicológica $\left(U=4756.5, Z=-4.33, p=0.00, r_{\text {bis }}\right.$ $=-0.27)$ y física leve $\left(U=5239.5, Z=-3.53, p=0.00, r_{\text {bis }}=-0.22\right)$ contra sus parejas; del mismo modo que ellas manifestaron haber sufrido con mayor frecuencia violencia física leve $(U=5678, Z=$ $-2.75, p=0.006, r_{\text {bis }}=-0.17$ ) por parte de sus parejas.

\section{Asociaciones entre violencia filioparental}

Los resultados indicaron que en la muestra de mujeres se presentó una vinculación entre la VFP psicológica contra el padre y la VFP psicológica contra la madre $\left(r_{h o}=.586, n=174, p=.01\right)$, al igual que entre la VFP física contra el padre y la VFP física contra la madre $\left(r_{h o}=.436, n=174, p=.01\right)$. En cuanto a la muestra de hombres, se 
encontró asociación entre la VFP psicológica contra el padre y la VFP psicológica contra la madre $\left(r_{h o}=.681, n=82, p=.01\right)$, y también entre la VFP física contra el padre y la VFP psicológica contra el padre $\left(r_{h o}=.538, n=82, p=.01\right)$. (Ver Tabla 4).

\section{Asociaciones entre la violencia observada entre los padres}

Como se puede observar en la Tabla 4, en la muestra de mujeres se encontraron vinculaciones positivas y significativas, como la que asocia la violencia física leve perpetrada por el padre y la violencia psicológica perpetrada por el padre $\left(r_{h o}=.757, n=174, p=.01\right)$, la que relaciona la violencia física leve perpetrada por la madre y la violencia psicológica perpetrada por la madre $\left(r_{h o}=.682, n=174\right.$, $p=.01$ ), e igualmente resalta el vínculo que hay entre la violencia psicológica perpetrada por el padre y la violencia psicológica perpetrada por la madre $\left(r_{h o}=.615, n=174, p=.01\right)$. Respecto a la muestra de hombres, también se hallaron muchas asociaciones altas y significativas, pero por razones de espacio se destacarán solo las más altas. Así, en primer lugar, se puede mencionar la asociación entre la violencia psicológica perpetrada por el padre y la violencia física leve perpetrada por el padre $\left(r_{h o}=.813, n=82\right.$, $p=.01$ ), en segundo lugar, el vínculo entre la violencia psicológica perpetrada por la madre y la violencia física leve perpetrada por la madre $\left(r_{h o}=.697, n=82, p=.01\right)$, y en tercer lugar, la relación entre la violencia psicológica perpetrada por la madre y la violencia psicológica cometida por el padre $\left(r_{h o}=.684, n=82, p=.01\right)$.

\section{Asociaciones entre la violencia cometida y sufrida en las relacio- nes de pareja}

En cuanto a la violencia cometida y sufrida, los resultados hallados en la muestra de mujeres señalaron índices de correlación significativos entre la violencia psicológica tanto cometida como sufrida $\left(r_{h o}=.741, n=174, p=.01\right)$, al igual que entre la violencia física leve cometida y sufrida $\left(r_{h o}=.726, n=174, p=.01\right)$. Y en la muestra de hombres, se encontraron asociaciones altas y significativas,

Tabla 1. Estadísticos descriptivos por sexo

\begin{tabular}{|c|c|c|c|c|c|c|}
\hline & \multicolumn{2}{|c|}{$\alpha$} & \multicolumn{2}{|c|}{$\bar{x}$} & \multicolumn{2}{|c|}{$D T$} \\
\hline & M & $\mathrm{H}$ & $\mathrm{M}$ & $\mathrm{H}$ & $\mathrm{M}$ & $\mathrm{H}$ \\
\hline VFP física hacia la madre & 0.75 & 0.97 & 2.26 & 1.95 & 0.91 & 0.77 \\
\hline VFP psicológica hacia la madre & 0.77 & 0.82 & 1.47 & 1.33 & 0.62 & 0.51 \\
\hline VFP física hacia el padre & 0.66 & 0.92 & 1.05 & 1.04 & 0.24 & 0.33 \\
\hline VFP psicológica hacia el padre & 0.81 & 0.87 & 0.04 & 0.05 & 0.21 & 0.34 \\
\hline Violencia psicológica cometida por la madre & 0.76 & 0.72 & 0.58 & 0.45 & 0.48 & 0.49 \\
\hline Violencia física leve cometida por la madre & 0.89 & 0.86 & 2.00 & 1.91 & 0.86 & 1.02 \\
\hline Violencia física grave cometida por la madre & 0.90 & 0.88 & 1.41 & 1.34 & 0.68 & 0.67 \\
\hline Violencia psicológica cometida por el padre & 0.75 & 0.85 & 1.07 & 1.07 & 0.29 & 0.46 \\
\hline Violencia física leve cometida por el padre & 0.91 & 0.92 & 0.05 & 0.17 & 0.21 & 0.57 \\
\hline Violencia física grave cometida por el padre & 0.87 & 0.97 & 0.43 & 0.43 & 0.48 & 0.59 \\
\hline Violencia psicológica cometida & 0.74 & 0.67 & 2.04 & 1.63 & 0.82 & 0.68 \\
\hline Violencia psicológica sufrida & 0.66 & 0.78 & 1.78 & 1.78 & 0.67 & 0.81 \\
\hline Violencia física leve cometida & 0.89 & 0.92 & 1.31 & 1.20 & 0.50 & 0.50 \\
\hline Violencia física leve sufrida & 0.90 & 0.95 & 1.28 & 1.23 & 0.50 & 0.60 \\
\hline Violencia física grave cometida & 0.62 & 1.00 & 1.04 & 1.05 & 0.21 & 0.44 \\
\hline Violencia física grave sufrida & 0.69 & 0.99 & 1.05 & 1.06 & 0.26 & 0.44 \\
\hline
\end{tabular}

Nota. $\alpha=$ Alfa de Cronbach, $\bar{x}=$ Media, $D T=$ Desviación Típica, $M=$ Mujeres $(n=174)$, H=Hombres $(n=82)$.

Tabla 2. Incidencia de la violencia filioparental en la muestra total $(n=256)$

\begin{tabular}{lcccc}
\hline & $\bar{x}$ & $D T$ & \multicolumn{2}{c}{$\begin{array}{c}\text { Al menos una vez } \\
\mathrm{n}(\%)\end{array}$} \\
\hline VFP psicológica hacia la madre & 0.54 & 0.48 & $41(16 \%)$ & $215(85 \%)$ \\
VFP psicológica hacia el padre & 0.43 & 0.52 & $64(25 \%)$ & $192(76 \%)$ \\
VFP física hacia la madre & 0.04 & 0.26 & $241(95 \%)$ & $15(6 \%)$ \\
VFP física hacia el padre & 0.09 & 0.37 & $228(90 \%)$ & $28(11 \%)$ \\
\hline
\end{tabular}

Nota. $\bar{x}=$ Media, $D T=$ Desviación Típica. 
como lo son la existente entre la violencia psicológica cometida y sufrida ( $\left.r_{h_{0}}=.850, n=82, p=.01\right)$, y el vínculo entre la violencia física leve cometida y sufrida $\left(r_{h o}=.850, n=82, p=.01\right)$.

\section{Asociaciones entre las diversas violencias}

Por último, se encontraron asociaciones significativas entre los diversos tipos de violencia, como en el caso de la muestra de mujeres, donde se hallaron índices de correlación significativos entre la VFP psicológica contra el padre y la violencia física leve perpetrada por el padre $\left(r_{h o}=.518, n=174, p=.01\right)$, al igual que entre la VFP psicológica contra el padre y la violencia psicológica perpetrada por el padre $\left(r_{h o}=.493, n=174, p=.01\right)$. En cuanto a la muestra de hombres, se obtuvieron correlaciones significativas entre la violencia física grave sufrida y la violencia física grave perpetrada por la madre $\left(r_{h o}=.817, n=82, p=.01\right)$, entre la violencia física grave cometida y la violencia física grave perpetrada por la madre $\left(r_{n o}=.711, n=82\right.$, $p=.01)$, y entre la violencia física grave perpetrada por la madre y la VFP física contra la madre $\left(r_{h o}=.707, n=82, p=.01\right)$.

Tabla 3. Diferencias entre sexos en las variables estudiadas

\begin{tabular}{|c|c|c|c|c|c|c|c|c|c|c|}
\hline & & $n$ & $\bar{x}$ & $D T$ & $M d$ & Rango & $U$ & $Z$ & $p$ & $r_{\text {bis }}$ \\
\hline \multirow{2}{*}{ VFP física hacia la madre } & M & 174 & 2.26 & 0.91 & 0 & 129.1 & \multirow{2}{*}{7030.5} & \multirow{2}{*}{-0.46} & \multirow{2}{*}{0.64} & \multirow{2}{*}{-0.02} \\
\hline & $\mathrm{H}$ & 82 & 1.95 & 0.77 & 0 & 127.2 & & & & \\
\hline \multirow{2}{*}{ VFP psicológica hacia la madre } & M & 174 & 1.47 & 0.62 & 0.42 & 136.1 & \multirow{2}{*}{5806} & \multirow{2}{*}{-2.41} & \multirow{2}{*}{0.01} & \multirow{2}{*}{-0.15} \\
\hline & $\mathrm{H}$ & 82 & 1.33 & 0.51 & 0.28 & 112.3 & & & & \\
\hline \multirow{2}{*}{ VFP física hacia el padre } & M & 174 & 1.05 & 0.24 & 0 & 125.4 & \multirow{2}{*}{6596} & \multirow{2}{*}{-1.79} & \multirow{2}{*}{0.07} & \multirow{2}{*}{-0.11} \\
\hline & $\mathrm{H}$ & 82 & 1.04 & 0.33 & 0 & 135.1 & & & & \\
\hline \multirow{2}{*}{ VFP psicológica hacia el padre } & M & 174 & 0.04 & 0.21 & 0.28 & 132.48 & \multirow{2}{*}{6441} & \multirow{2}{*}{-1.27} & \multirow{2}{*}{0.2} & \multirow{2}{*}{-0.07} \\
\hline & $\mathrm{H}$ & 82 & 0.05 & 0.34 & 0.28 & 120.1 & & & & \\
\hline \multirow{2}{*}{ Violencia psicológica cometida por la madre } & M & 174 & 0.58 & 0.48 & 2.25 & 136.3 & \multirow{2}{*}{5777.5} & \multirow{2}{*}{-2.46} & \multirow{2}{*}{0.01} & \multirow{2}{*}{-0.15} \\
\hline & $\mathrm{H}$ & 82 & 0.45 & 0.49 & 1.87 & 112.0 & & & & \\
\hline \multirow{2}{*}{ Violencia física leve cometida por la madre } & M & 174 & 2 & 0.86 & 1.25 & 135.6 & \multirow{2}{*}{5893.5} & & & \\
\hline & $\mathrm{H}$ & 82 & 1.91 & 1.02 & 1.12 & 113.4 & & -2.28 & 0.02 & -0.14 \\
\hline & M & 174 & 1.41 & 0.68 & 1 & 130.1 & & & & \\
\hline Violencia tisıca grave cometida por la madre & $\mathrm{H}$ & 82 & 1.34 & 0.67 & 1 & 125.2 & 6860 & -1.3 & 0.19 & -0.08 \\
\hline Violencia ncicolóqica cometida nor l nadre & $M$ & 174 & 1.07 & 0.29 & 2 & 133.1 & & 1,16 & 19 & ח ח 0 \\
\hline 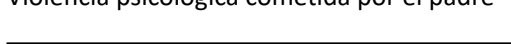 & $\mathrm{H}$ & 82 & 1.07 & 0.46 & 1.5 & 118.7 & 0כ2 & -1.40 & 0.14 & -0.09 \\
\hline Wiglancin f'sina lousomotidanor hadro & $M$ & 174 & 0.05 & 0.21 & 1.12 & 133.7 & ז 2 & 177 & 000 & 01 \\
\hline violencid isicd leve cometiad por el paure & $\mathrm{H}$ & 82 & 0.17 & 0.57 & 1 & 117.4 & $0 \angle 2 \angle . J$ & -1.12 & 0.00 & -0.1 \\
\hline & $M$ & 174 & 0.43 & 0.48 & 1 & 129.6 & & & & \\
\hline violencia nsica grave comenaa por el paare & $\mathrm{H}$ & 82 & 0.43 & 0.59 & 1 & 126.3 & 6951 & -0.16 & 0.44 & -0.04 \\
\hline & $M$ & 174 & 2.04 & 0.82 & 2 & 142.2 & & & & \\
\hline Violencia psicológica cometida en la pareja & $\mathrm{H}$ & 82 & 1.63 & 0.68 & 1.5 & 99.5 & 4756.5 & -4.33 & 0 & -0.27 \\
\hline Viploncia ncicológicz cufrida on la noroia & $M$ & 174 & 1.78 & 0.67 & 1.75 & 130.4 & 7075 & 061 & 052 & בח 2 \\
\hline violencia psicologica sutrida en la pareja & $\mathrm{H}$ & 82 & 1.78 & 0.81 & 1.5 & 124.4 & $0 / 91.5$ & -0.61 & 0.53 & -0.03 \\
\hline 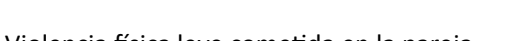 & $M$ & 174 & 1.31 & 0.5 & 1.12 & 139.4 & & 2 & 0 & 27 \\
\hline violencid issicd reve comeciud ent ld parejd & $\mathrm{H}$ & 82 & 1.2 & 0.5 & 1.06 & 105.4 & 5239.5 & -3.53 & 0 & -0.22 \\
\hline 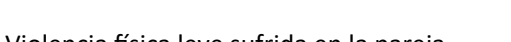 & $M$ & 174 & 1.28 & 0.5 & 1.12 & 136.9 & 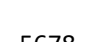 & & & \\
\hline violencia nsica reve sutrida en ra pareja & $\mathrm{H}$ & 82 & 1.23 & 0.6 & 1.06 & 110.7 & $56 / 8$ & -2.15 & 0.01 & $-0.1 /$ \\
\hline & $M$ & 174 & 1.04 & 0.21 & 1 & 129.9 & & & & \\
\hline Vıolencıa fisıca grave cometida en la pareja & $\mathrm{H}$ & 82 & 1.05 & 0.44 & 1 & 125.6 & $689 /$ & -1.34 & $0.1 /$ & -0.08 \\
\hline & $M$ & 174 & 1.05 & 0.26 & 1 & 128.7 & & 011 & 000 & 0 \\
\hline & $\mathrm{H}$ & 82 & 1.06 & 0.44 & 1 & 128.2 & 1100.3 & -0.14 & 0.80 & U \\
\hline
\end{tabular}

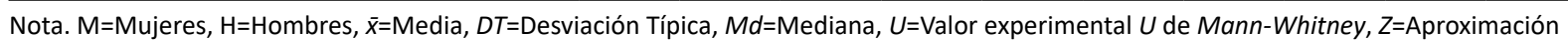
por la Normal, $p=$ Significación, $r_{\text {bis }}=r$ de Rosenthal (tamaño del efecto). 


\section{DISCUSIÓN}

El objetivo de esta investigación fue estudiar la frecuencia de violencia filioparental y su co-ocurrencia con la violencia en las relaciones de pareja y la observada entre los padres y la co-ocurrencia entre estos fenómenos, en alumnado de una universidad pública del sureste de México; para ello se han planteado hipótesis específicas que se discuten a continuación.

Con referencia a la primera hipótesis planteada en este estudio, se esperaba que la VFP fuera más frecuente hacia las madres, tal y como lo han sugerido algunos estudios (Calvete, Gámez-Guadix, y Orue, 2014; Calvete, Orue y González-Cabrera, 2017). No obstante lo anterior solo se hizo evidente en los resultados obtenidos para la VFP psicológica, ya que la VFP física la padecieron mayormente los padres, concordando en ambas cuestiones con lo hallado por Vázquez-Sánchez et al. (2019), al igual que con Álvarez, Sepúlveda y Espinoza (2016). Por otra parte, los datos contrastaron con lo encontrado por Ibabe (2015), quien no halló diferencias significativas en la perpetración de violencia física en función del sexo de los progenitores, mientras que Calvete y Veytia (2018) encontraron una mayor incidencia en todos los tipos de violencia (psicológica, psicológica severa, física y física severa) hacia la ma- dre. La presencia de VFP contra la madre se podría explicar por el hecho de que las madres son quienes habitualmente asumen el rol de la crianza de los hijos, suelen pasar más tiempo a solas con ellos y pueden ser percibidas como débiles (Martínez et al., 2015). En cuanto a la segunda hipótesis, "las mujeres ejercen más frecuentemente la VFP psicológica y los hombres la VFP física" (Lozano, Estévez y Carballo, 2013), dicha diferenciación únicamente se manifestó en la VFP psicológica contra la madre, cuya prevalencia fue mayor en mujeres. En cambio, acorde con el resto de las subescalas de VFP no hubo diferencias significativas entre sexos, coincidiendo con lo señalado Aroca-Montolío, Lorenzo-Moledo y Miró-Pérez (2014). Tales resultados difieren con lo encontrado por Ibabe, Jaureguizar y Bentler (2013), quienes hallaron que los hijos dirigieron más VFP física hacia sus padres que las hijas. Estos resultados podrían ser explicados desde la teoría intergeneracional de la violencia, según la cual la observación o sufrimiento de malos tratos en el contexto familiar son un factor de riesgo para los hijos, posibilitando el aprendizaje de conductas tanto pasivas (ser víctima) como violentas (ser agresor) que podrían ejercerse en el futuro (Molla-Esparza y Aroca-Montolío, 2018). En apoyo a lo anterior, en la presente investigación se encontraron correla-

Tabla 4. Correlaciones entre violencia filioparental, violencia observada entre los padres y violencia en las relaciones de pareja, en hombres y mujeres

\begin{tabular}{|c|c|c|c|c|c|c|c|c|c|c|c|c|c|c|c|c|c|}
\hline \multicolumn{18}{|c|}{ HOMBRES } \\
\hline \multirow{17}{*}{$\begin{array}{l}\text { 岃 } \\
\stackrel{\sim}{\breve{Z}} \\
\stackrel{\Sigma}{\Sigma}\end{array}$} & & 1 & 2 & 3 & 4 & 5 & 6 & 7 & 8 & 9 & 10 & 11 & 12 & 13 & 14 & 15 & 16 \\
\hline & 1 & - & 0.167 & $.238^{*}$ & 0.144 & 0.176 & $.221^{*}$ & $.707^{* *}$ & $.248^{*}$ & 0.116 & $.231^{*}$ & $.222^{*}$ & 0.198 & 0.179 & 0.184 & $.509^{* *}$ & $.570^{* *}$ \\
\hline & 2 & $.250^{* *}$ & - & $.363^{* *}$ & $.681^{* *}$ & $.464^{* *}$ & $.479^{* *}$ & $.263^{*}$ & $.495^{* *}$ & $.356^{* *}$ & $.223^{*}$ & $.348^{* *}$ & $.287^{* *}$ & $.261^{*}$ & $.285^{* *}$ & 0.192 & $.284^{* *}$ \\
\hline & 3 & $.436^{* *}$ & $.206^{* *}$ & - & $.538^{* *}$ & $.330^{* *}$ & $.469^{* *}$ & $.394^{* *}$ & $.459^{* *}$ & $.566^{* *}$ & $.562^{* *}$ & 0.108 & 0.064 & 0.192 & 0.166 & $.295^{* *}$ & $.298^{* *}$ \\
\hline & 4 & $.203^{* *}$ & $.586^{* *}$ & $.380^{* *}$ & - & $.416^{* *}$ & $.469^{* *}$ & $.274^{*}$ & $.577^{* *}$ & $.549^{* *}$ & $.266^{*}$ & $.238^{*}$ & $.247^{*}$ & $.303^{* *}$ & $.360^{* *}$ & 0.195 & $.305^{* *}$ \\
\hline & 5 & 0.139 & $.330^{* *}$ & $.197^{* *}$ & $.283^{* *}$ & - & $.697^{* *}$ & 0.09 & $.684^{* *}$ & $.616^{* *}$ & 0.142 & $.364^{* *}$ & $.333^{* *}$ & $.277^{*}$ & $.253^{*}$ & -0.06 & 0.128 \\
\hline & 6 & $.325^{* *}$ & $.426^{* *}$ & $.278^{* *}$ & $.317^{* *}$ & $.682^{* *}$ & - & $.252^{*}$ & $.609^{* *}$ & $.646^{* *}$ & $.273^{*}$ & $.364^{* *}$ & $.362^{* *}$ & $.377^{* *}$ & $.335^{* *}$ & 0.185 & $.239^{*}$ \\
\hline & 7 & $.419^{* *}$ & 0.091 & $.177^{*}$ & 0.121 & $.254^{* *}$ & $.374^{* *}$ & - & 0.189 & 0.201 & .349** & $.269^{*}$ & $.264^{*}$ & $.288^{* *}$ & $.286^{* *}$ & $.711^{* *}$ & $.817^{* *}$ \\
\hline & 8 & 0.13 & $.321^{* *}$ & $.313^{* *}$ & $.493^{* *}$ & $.615^{* *}$ & $.499^{* *}$ & 0.088 & - & $.813^{* *}$ & $.294^{* *}$ & $.265^{*}$ & $.232^{*}$ & 0.195 & $.266^{*}$ & 0.194 & 0.197 \\
\hline & 9 & 0.137 & $.279^{* *}$ & $.351^{* *}$ & $.518^{* *}$ & $.549^{* *}$ & $.566^{* *}$ & $.177^{*}$ & $.757^{* *}$ & - & $.405^{* *}$ & $.253^{*}$ & $.221^{*}$ & $.270^{*}$ & $.288^{* *}$ & 0.207 & 0.21 \\
\hline & 10 & $.197^{* *}$ & 0.072 & $.293^{* *}$ & 0.126 & $.333^{* *}$ & $.375^{* *}$ & $.293^{* *}$ & $.370^{* *}$ & $.437^{* *}$ & - & 0.09 & 0.068 & $.225^{*}$ & $.224^{*}$ & $.509^{* *}$ & $.276^{*}$ \\
\hline & 11 & $.199^{* *}$ & $.356^{* *}$ & 0.113 & $.275^{* *}$ & $.259^{* *}$ & $.337^{* *}$ & 0.085 & $.327^{* *}$ & $.295^{* *}$ & 0.062 & - & $.850^{* *}$ & $.581^{* *}$ & $.594^{* *}$ & 0.193 & $.328^{* *}$ \\
\hline & 12 & $.174^{*}$ & $.369^{* *}$ & 0.092 & $.325^{* *}$ & $.216^{* *}$ & $.332^{* *}$ & $.167^{*}$ & $.283^{* *}$ & $.245^{* *}$ & 0.098 & $.741^{* *}$ & - & $.514^{* *}$ & $.601^{* *}$ & 0.192 & $.321^{* *}$ \\
\hline & 13 & $.221^{* *}$ & $.472^{* *}$ & 0.134 & $.402^{* *}$ & $.193^{*}$ & $.312^{* *}$ & 0.13 & $.179^{*}$ & $.185^{*}$ & 0.142 & $.656^{* *}$ & $.541^{* *}$ & - & $.850^{* *}$ & 0.205 & $.347^{* *}$ \\
\hline & 14 & $.211^{* *}$ & $.338^{* *}$ & 0.114 & $.290^{* *}$ & $.228^{* *}$ & $.277^{* *}$ & $.163^{*}$ & $.179^{*}$ & $.259^{* *}$ & 0.128 & $.591^{* *}$ & $.561^{* *}$ & $.726^{* *}$ & - & 0.206 & $.339^{* *}$ \\
\hline & 15 & $.303^{* *}$ & $.194^{*}$ & 0.147 & 0.092 & 0.055 & $.166^{*}$ & $.178^{*}$ & 0.073 & 0.092 & 0.148 & $.213^{* *}$ & $.245^{* *}$ & $.311^{* *}$ & $.315^{* *}$ & - & $.585^{* *}$ \\
\hline & 16 & $.204^{* *}$ & 0.048 & 0.052 & -0.081 & -0.08 & 0.065 & $.194^{*}$ & -0.1 & -0.08 & 0.063 & $.159^{*}$ & $.186^{*}$ & $.278^{* *}$ & $.317^{* *}$ & $.530 * *$ & - \\
\hline
\end{tabular}

Nota. **. La correlación es significativa en el nivel 0,01 (2 colas), *. La correlación es significativa en el nivel 0,05 (2 colas).

1=Violencia filioparental física hacia la madre; 2=Violencia filioparental psicológica hacia la madre; 3=Violencia filioparental física hacia el padre; 4=Violencia filioparental psicológica hacia el padre; 5=Violencia psicológica cometida por la madre; 6=Violencia física leve cometida por la madre; 7=Violencia física grave cometida por la madre; 8=Violencia psicológica cometida por el padre; 9=Violencia física leve cometida por el padre; 10=Violencia física grave cometida por el padre; 11=Violencia psicológica cometida; 12=Violencia psicológica sufrida; 13=Violencia física leve cometida; 14=Violencia física leve sufrida; 15=Violencia física grave cometida y 16=Violencia física grave sufrida. 
ciones entre la VFP psicológica contra el padre y la violencia física leve perpetrada por el padre, al igual que entre la VFP psicológica contra el padre y la violencia psicológica perpetrada por el padre en las hijas. Asimismo, se halló una correlación significativa entre la violencia física grave perpetrada por la madre y la VFP física contra la madre en los hijos.

Con respecto a la tercera hipótesis, se esperaba que las mujeres ejercieran con mayor frecuencia la violencia psicológica, y los hombres la violencia física (Alegría y Rodríguez, 2015). Pues bien, los resultados señalaron diferencias significativas entre sexos con las mujeres superando a los hombres, reafirmando la primera parte de la tercera suposición y concordando con lo mencionado por Rubio-Garay et al., (2017). Sin embargo, no se pudo ratificar que los hombres ejercieran con mayor frecuencia la violencia física y, al contrario, como ya se ha señalado en diversos estudios (Cortés-Ayala et al., 2015; Marasca y Falcke, 2015; Martínez, Vargas y Novoa, 2016; Nava-Reyes et al., 2018; Peña et al., 2018; Rodríguez, Riosvelasco y Castillo, 2018; Wincentak, Connolly y Card, 2017), las mujeres perpetraron en mayor medida la violencia física. De acuerdo con Gracia-Leiva (2019), una de los tópicos más controvertidos de la violencia en el noviazgo son las diferencias por sexos en la prevalencia tanto de perpetración como de victimización, ya que algunas investigaciones señalan con más frecuencia a los hombres como agresores, otros a las mujeres, y unos más indican altas tasas de bidireccionalidad; además si hallan diferencias, o si encuentran tasas mayores de violencia en mujeres, la magnitud estadística de la diferencia es pequeña, siendo una posible causa el hecho de que tales trabajos no toman en cuenta las tasas de subestimación de la violencia por parte de las mujeres. Por otra parte, algunas pesquisas han resaltado que los hombres suelen rechazar menos la violencia, por lo que la justifican más que las mujeres; y de igual forma varios autores han señalado el hecho de que los hombres tienden más a legitimizar la violencia como respuesta, quitándole importancia, mientras que las mujeres sobrevaloran sus actos, provocando que se sientan culpables por ellos (Pazos, Oliva y Hernando, 2014). No sobra decir que, afortunadamente, la frecuencia en la población de la violencia cometida y sufrida encontrada fue baja, resultados que concuerdan con hallazgos como los de Celis-Sauce y Rojas-Solís (2015).

Por otra parte, se esperaba corroborar que el haber sufrido algún tipo de violencia en la pareja se asociaría con la perpetración de la misma, es decir que la violencia de pareja se presentaría de manera bidireccional. En ese sentido, se obtuvieron correlaciones significativas entre la perpetración y victimización de la violencia psicológica, al igual que de la física leve, corroborando así el cuarto supuesto. En ese tenor, es pertinente recordar que en la presente investigación tanto en la muestra de mujeres como en la de hombres se encontraron correlaciones significativas entre la violencia psicológica cometida y sufrida, al igual que entre la violencia física leve cometida y sufrida, coincidiendo con Celis-Sauce y Rojas-Solís (2015). Es así como se hizo evidente la bidireccionalidad del fenómeno, ya presente en múltiples estudios (Cortés-Aya- la et al., 2015; Fernández-González, O’Leary y Muñoz-Rivas, 2013; Palmetto et al., 2013; Valdivia y González, 2014), confirmando el supuesto final.

Cabe mencionar que la bidireccionalidad también se halló en el vínculo obtenido entre la violencia psicológica observada entre los padres, pues en los hombres se observó una correlación positiva entre la violencia física grave perpetrada por la madre y la violencia física grave cometida contra la pareja.

Por otro lado, y siguiendo lo sugerido por otros autores (Ibabe, Arnoso y Elgorriaga, 2020; Pacheco, 2015) respecto a que quienes hayan observado o presenciado violencia entre sus progenitores podrían presentar conductas violentas en sus relaciones de noviazgo, se encontró correlación entre la violencia física grave perpetrada por la madre y violencia física grave sufrida en el noviazgo. Entre otras razones, esto podría suceder como resultado de lo vivido en la dinámica familiar, de tal manera que se permite este tipo de violencia en las relaciones de pareja tras una normalización y aceptación como medio de resolver conflictos, (de Alencar-Rodrigues y Cantera, 2012), por lo que la exposición a la violencia podría ser un predictor de la violencia en el noviazgo (Bonilla-Algovia y Rivas-Rivero, 2019).

\section{Limitaciones y fortalezas}

Dentro de las limitaciones metodológicas de esta investigación se encuentran el tipo de muestreo empleado (no probabilístico) y la desproporción entre la cantidad de mujeres y hombres participantes, añadido a ello es preciso indicar que tanto el CTS como el M-CTS no son instrumentos destinados íntegramente a la evaluación de violencia, sino más bien tácticas de solución de conflictos, por lo que los resultados derivados de ellos requieren cautela al asociarse a violencia cometida o sufrida.

\section{Futuras líneas de investigación}

Los autores sugieren indagar más respecto a la VFP, pues como se ha referido anteriormente se trata de un campo en el cual aún hay mucho por explorar, especialmente con sus características actuales (Molla-Esparza y Aroca-Montolío, 2018). Y si bien la violencia en las relaciones de pareja es un tópico mucho más abordado, su evolución es constante, especialmente cuando siguen surgiendo otro tipo de relaciones de pareja (free, amigovio, etc.), por lo que resultaría pertinente continuar investigando tal fenómeno; sin olvidar la necesaria inclusión de otro tipo de poblaciones como parejas del mismo sexo, provenientes de zonas rurales, no escolarizadas o indígenas.

\section{Conclusiones}

Para finalizar, los resultados obtenidos pretenden abonar en el corpus empírico sobre la co-ocurrencia de distintas formas de violencia en adolescentes, especialmente en jóvenes tabasqueños; entre otras implicaciones, resalta el hecho de la violencia filioparental (psicológica y física), al igual que contra la pareja (psicológica, física leve y, en menor medida, física grave) se está haciendo evidente tanto en mujeres como hombres, por lo que, además, 
parecen contar con un carácter bidireccional tanto en los participantes como en sus progenitores. Estos datos, con las debidas precauciones, señalan la necesidad de implementar programas de prevención e intervención en la violencia en todos los ámbitos $y$, en especial, evitar seguir fragmentando el estudio de la violencia interpersonal e intrafamiliar.

\section{ORCID}

Daniela Cancino-Padilla https://orcid.org/0000-0001-8430-7218

José Luis Rojas-Solís http://orcid.org/0000-0001-6339-4607

\section{CONFLICTO DE INTERÉS}

Sin conflicto de interés.

\section{FINANCIAMIENTO}

Autofinanciado.

\section{AGRADECIMIENTOS}

La alumna Daniela Cancino-Padilla realizó parte de este artículo dentro del Verano de la Investigación Científica y Tecnológica del Pacífico, Programa Delfín; el alumno Christian Alexis Romero-Méndez realizó parte de este manuscrito dentro del Verano de la Investigación Científica - Academia Mexicana de Ciencias Estudio realizado dentro del Cuerpo Académico (BUAP-CA-330): "Prevención de la violencia: Educando para una Cultura de Paz a través de la Participación Social".

\section{REFERENCIAS}

Alegría, M., \& Rodríguez, A. (2015). Violencia en el noviazgo: perpetración, victimización y violencia mutua. Una revisión. Actualidades en Psicología, 29(118), 57-72. http://dx.doi.org/10.15517/ap.v29i118.16008

Alvarado, G. P. A. A. (2015). Transmisión transgeneracional de la violencia de pareja y funcionalidad familiar de hombres y mujeres de la ciudad de Trujillo. In Crescendo, 6(2), 11-21. https://doi.org/10.21895/incres.2015.v6n2.02.

Álvarez, A. A. J., Sepúlveda, G. R. E., \& Espinoza, M. S. M. (2016). Prevalencia de la violencia filio-parental en adolescentes de la ciudad de Osorno. Pensamiento y Acción Interdisciplinaria, 1(1), 59-74. Recuperado de: http://revistapai.ucm.cl/ article/view/156/151

Aroca-Montolío, C., Lorenzo-Moledo, M., \& Miró-Pérez, C. (2014). La violencia filio-parental: un análisis de sus claves. Anales de Psicología, 30(1) 157-170. https://doi.org/10.6018/analesps.30.1.149521

Bandura, A. (1982). Teoría del Aprendizaje Social. Madrid: Espasa Universitaria.

Batiza, A. F. J. (2017). La violencia de pareja: Un enemigo silencioso. Archivos de Criminología, Seguridad Privada y Criminalística, 18, 144-151. Recuperado de: https://dialnet.unirioja.es/servlet/articulo?codigo $=5813533$

Bayona, L., Chivita, A. V. \& Gaitan, D. C. (2015). Violencia de pareja y construcción de discurso sobre la subjetividad femenina. Informes Psicológicos, 15(1), 127 143. http://dx.doi.org/10.18566/infpsicv15n1a07

Bolívar, S. Y., Rey, A. C. A. \& Martínez, G. J. A. (2017). Funcionalidad familiar, número de relaciones y maltrato en el noviazgo en estudiantes de secundaria. Psicología desde el Caribe, 34(1). Recuperado de: https://dialnet.unirioja.es/servlet/ articulo?codigo $=6552639$

Bonilla-Algovia, E., \& Rivas-Rivero, E. (2019). Violencia en el noviazgo en estudiantes colombianos: relación con la violencia de género en el entorno. Interacciones, 5(3). http://dx.doi.org/10.24016/2019.v5n3.197

Calvete, E., Gámez-Guadix, M., \& Orue, I. (2014). Características familiares asociadas a violencia filio-parental en adolescentes. Anales de psicología, 30(3), 11761182. http://dx.doi.org/10.6018/analesps.30.3.166291

Calvete, E., Gámez-Guadix, M., Orue, I., González-Diez, Z., de Arroyabe, E. L.,
Sampedro, R., ..., \& Borrajo, E. (2013). Brief report: The Adolescent Child-to-Parent Aggression Questionnaire: An examination of aggressions against parents in Spanish adolescents. Journal of Adolescence, 36(6), 1077-1081. https://doi. org/10.1016/j.adolescence.2013.08.017

Calvete, E., Orue, I., \& González-Cabrera, J. (2017). Violencia filio parental: comparando lo que informan los adolescentes y sus progenitores. Revista de Psicología Clínica con Niños y Adolescentes, 4(1), 9-15. Recuperado de: https:// dialnet.unirioja.es/servlet/articulo?codigo $=5789314$

Calvete, E., \& Veytia, M. (2018). Adaptación del Cuestionario de Violencia Filio-Parental en Adolescentes Mexicanos. Revista Latinoamericana de Psicología, 50(1), 49-59. http://dx.doi.org/10.14349/rlp.2018.v50.n1.5.

Caridade, S., Braga, T. \& Borrajo, E. (2019). Cyber dating abuse (CDA): Evidence from a systematic review. Aggression and Violent Behavior, 48, 152-168. https:// doi.org/10.1016/j.avb.2019.08.018

Celis-Sauce, A., \& Rojas-Solís, J. L. (2015). Adolescentes mexicanos como víctimas y perpetradores de violencia en el noviazgo. Reidocrea, 4, 60-65. Recuperado de: http://hdl.handle.net/10481/35150

Cohen, J.W. (1988). Statistical power analysis for the behavioral sciences (2nd edn). Hillsdale, NJ: Lawrence Erlbaum Associates.

Cortés-Ayala, L., Flores, M., Bringas, C., Rodríguez-Franco, L., López-Cepero, J., \& Rodríguez, F. J. (2015). Relación de maltrato en el noviazgo de jóvenes mexicanos: análisis diferencial por sexo y nivel de estudios. Terapia psicológica, 33(1) 5-12. http://dx.doi.org/10.4067/S0718-48082015000100001

Dardis, C. M., Dixon, K. J., Edwards, K. M., \& Turchik, J. A. (2015). An examination of the factors related to dating violence perpetration among young men and women and associated theoretical explanations: A review of the literature. Trauma, Violence, \& Abuse, 16(2), 136-152. https://doi.org/10.1177/1524838013517559 de Alencar-Rodrigues, R., \& Cantera, L. (2012). Violencia de género en la pareja: Una revisión teórica. Psico, 41(1), 116-126. Recuperado de: https://ddd.uab.cat/ record/130820? In=es

del Moral, A. G., Martínez, F. B., Suárez, R. C., Ávila, G. M. E., \& Vera, J. J. A. (2015) Teorías sobre el inicio de la violencia filio-parental desde la perspectiva parental: un estudio exploratorio. Pensamiento Psicológico, 13(2), 95-107. http://dx.doi. org/10.11144/Javerianacali.PPSI13-2.tivf

Eynon, R., Schroeder, R. y Fry, J. (2009) New techniques in online research: Challenges for research ethics, Twenty-First Century Society, 4(2), 187-199. https:// doi.org/10.1080/17450140903000308

Espinobarros-Nava, F., Muñoz-Ponce, N. N., \& Rojas-Solís, J. L. (2018). Coocurrencia de distintas violencias en el noviazgo en una muestra de jóvenes mexicanos procedentes de zona rural. Summa Psicológica UST, 15(2) 154-161. Recuperado de: https://dialnet.unirioja.es/servlet/articulo?codigo=7009160

Fernández-González, L., O'Leary, K. D., \& Muñoz-Rivas, M. J. (2013). We Are Not Joking: Need for controls in reports of dating violence. Journal of Interpersonal Violence, 28(3), 602-620. https://doi.org/10.1177/0886260512455518

Gracia-Leiva, M., Puente-Martínez, A., Ubillos-Landa, S., \& Páez-Rovira, D. (2019). La violencia en el noviazgo (VN): una revisión de meta-análisis. Anales de Psicología, 35(2), 300-313. http://dx.doi.org/10.6018/analesps.35.2.333101

Ibabe, I. (2015). Family predictors of child-to-parent violence: the role of family discipline. Anales De Psicología, 31(2), 615-625. http://dx.doi.org/10.6018/analesps.31.2.174701

Ibabe, I., Arnoso, A., \& Elgorriaga, E. (2020). Child-to-Parent Violence as an Intervening Variable in the Relationship between Inter-Parental Violence Exposure and Dating Violence. International Journal of Environmental Research and Public Health, 17(5), 1514. https://doi.org/10.3390/ijerph17051514

Ibabe, I., Jaureguizar, J., \& Bentler, P. M. (2013). Risk factors for child-to-parent violence. Journal of family violence, 28(5), 523-534. https://doi.org/10.1007/ s10896-013-9512-2

Instituto Nacional de Estadística Geografía e Informática (2016). Encuesta Nacional sobre la Dinámica de las Relaciones en los Hogares (ENDIREH). México, D.F.: Autor.

Leen, E., Sorbring, E., Mawer, M., Holdsworth, E., Helsing, B. \& Bowen, E. (2013). Prevalence, dynamic risk factors and the efficacy of primary interventions for adolescentdating violence: An international review. Aggression and Violent Behavior, 18, 159-174. https://doi.org/10.1016/j.avb.2012.11.015

Llamazares, A., Vázquez, G. \& Zuñeda, A. (2013). Violencia filio-parental: propuesta de explicación desde un modelo procesual, Boletín de Psicología, 109, 85-99. 
Recuperado de: https://dialnet.unirioja.es/servlet/articulo?codigo=4495413 Lozano, S., Estévez, E. \& Carballo, J. L. (2013). Factores individuales y familiares de riesgo en casos de violencia filio-parental. Documentos de trabajo social: Revista de trabajo y acción social, 52, 239-254. Recuperado de: https://dialnet.unirioja. es/servlet/articulo?codigo $=4703109$

Makin-Byrd, K. \& Bierman, K. L. (2013). Individual and family predictors of the perpetration of dating violence and victimization in late adolescence. Journal of youth and adolescence, 42(4), 536-550. https://doi.org/10.1007/s10964-0129810-7

Marasca, A. R., \& Falcke, D. (2015). Forms of violence in the affective-sexual relationships of adolescents. Interpersona: An International Journal on Personal Relationships, 9(2), 200-214. http://dx.doi.org/10.23668/psycharchives.2210

Martínez G. J. A., Vargas G. R. \& Novoa G. M. (2016). Relación entre la violencia en el noviazgo y observación de modelos parentales de maltrato. Psychologia. Avances de la disciplina, 10(1),101-112. Recuperado de: http://www.scielo.org. co/scielo.php?script=sci_arttext\&pid=S1900-23862016000100010

Martínez, J. A., Vargas, R., \& Novoa, M. (2016). Relación entre la violencia en el noviazgo y observación de modelos parentales de maltrato. Psychologia. Avances de la disciplina 10(1), 101-112. Recuperado de: http://www.redalyc.org/articulo. oa?id $=297245905010$

Martínez, M. L., Estévez, E., Jiménez, T. I., \& Velilla, C. (2015). Child-parent violence: main characteristics, risk factors and keys to intervention. Papeles Del Psicólogo, 36(3), 216-224. Recuperado de: http://www.papelesdelpsicologo.es/ English/2615.pdf

Molla-Esparza, C., \& Aroca-Montolío, C. (2018). Menores que maltratan a sus progenitores: definición integral y su ciclo de violencia. Anuario de Psicología Jurídica, 28(1). https://doi.org/10.1016/j.apj.2017.01.001

Morales, D. N. E., \& Rodríguez, T. V. (2012). Experiencias de violencia en el noviazgo de mujeres en Puerto Rico. Revista puertorriqueña de psicología, 23, 5790. Recuperado de: http://pepsic.bvsalud.org/scielo.php?script=sci_arttext\&pi$\mathrm{d}=\mathrm{S} 1946-20262012000100003$

Muñoz-Rivas, M.J., Andreu, R. J. M., Graña, G. J. L., O'Leary, D. K. \& González, M.P. (2007). Validación de la versión modificada de la Conflicts Tactics Scale (M-CTS) en población juvenil española. Psicothema, 19(4), 692-697. Recuperado de: https://dialnet.unirioja.es/servlet/articulo?codigo $=2389574$

Nava-Reyes, M. A., Rojas-Solís, J. L., de la Paz Toldos-Romero, M., \& Morales-Quintero, L. A. (2018). Factores de género y violencia en el noviazgo de adolescentes. Boletín Cientifico Sapiens Research, 8(1), 54-70. Recuperado de: https://dialnet. unirioja.es/servlet/articulo?codigo $=6705582$

Nosek, B. A., Banaji, M. R., \& Greenwald, A. G. (2002). eResearch: Ethics, security, design, and control in psychological research on the Internet. Journal of Social Issues, 58, 161-176

Nunnaly, J. C. y Bernstein, I. H. (1994). Psychometric theory (3rd Ed.). New York, NJ: McGraw-Hill.

Ospina, M. \& Clavijo, K. A. (2016). Una mirada sistémica a la violencia de pareja: dinámica relacional, ¿̇configuradora del ciclo de violencia conyugal? Textos y Sentidos, 14, 105-122. Recuperado de: http://hdl.handle.net/10785/3504

Pacheco, V. M. J. (2015). Actitud hacia la violencia contra la mujer en la relación de pareja y el clima social familiar en adolescentes. Interacciones, 1(1), 29-44. http://dx.doi.org/10.24016/2015.v1n1.2

Palmetto, N., Davidson, L. L., Breitbart, V. \& Rickert, V. I. (2013). Predictors of physical intimate partner violence in the lives of young women: Victimization, perpetration, and bidirectional violence. Violence and Victims, 28(1), 103-121. http:// dx.doi.org/10.1891/0886-6708.28.1.103

Pazos, G. M., Oliva, D A., \& Hernando, G. Á. (2014). Violencia en relaciones de pareja de jóvenes y adolescentes. Revista latinoamericana de psicología, 46(3), 148-159. https://doi.org/10.1016/S0120-0534(14)70018-4

Peña, F., González B. Z., Sotelo K. V., Martínez J. I. V., Narváez Y. V., Rodríguez G. I. H., Parra, S. V. \& Ruíz R. L. (2018). Violencia en el noviazgo en jóvenes y adolescentes en la frontera norte de México. Journal Health NPEPS, 3(2), 426-440. http://dx.doi.org/10.30681/252610103117

Pereira, R., Loinaz, C. I., del Hoyo-Bilbao, J., Arrospide, J., Bertino, L., Calvo, A., ... \& Gutiérrez, M. M. (2017). Propuesta de definición de violencia filio-parental: Consenso de la Sociedad Española para el estudio de la Violencia Filio-Parental. Papeles del psicólogo, 38(3), 216-223. https://doi.org/10.23923/pap.psicol2017.2839
Rey-Anacona, C. A. (2013). Prevalencia y tipos de maltrato en el noviazgo en adolescentes y adultos jóvenes. Terapia psicológica, 31(2), 143-154. http://dx.doi. org/10.4067/S0718-48082013000200001

Rodríguez, H. R., Riosvelasco, M. L., \& Castillo, V. N. (2018). Violencia en el noviazgo, género y apoyo social en jóvenes universitarios. Escritos de psicología, 11(1), 1-9. http://dx.doi.org/10.5231/psy.writ.2018.2203

Rojas-Solís, J. L. (2013). Violencia en el noviazgo de universitarios en México: Una revisión. Revista Internacional de Psicología, 12(02). https://doi. org/10.33670/18181023.v12i02.71

Rojas-Solís, J. L., Vázquez-Aramburu, G., \& Llamazares-Rojo, J. A. (2016). Violencia filio-parental: una revisión de un fenómeno emergente en la investigación psicológica. Ajayu. 14(1), 140-161. Recuperado de: http://www.scielo.org.bo/scielo. php?pid=S2077-21612016000100007\&script=sci_arttext

Romo-Tobón, R. J., Vázquez-Sánchez, V., Rojas-Solís, J. L., \& Alvídrez, S. (2020). Cyberbullying y Ciberviolencia de pareja en alumnado de una universidad privada mexicana. Propósitos y representaciones, 8(2). http://dx.doi.org/10.20511/ pyr2020.v8n2.303

Ronzón-Tirado, R. C., Muñoz-Rivas, M. J., Zamarrón, M. D., \& Redondo, N. (2019). Cultural Adaptation of the Modified Version of the Conflicts Tactics Scale (M-CTS) in Mexican Adolescents. Frontiers in Psychology, 10, 619. https://doi. org/10.3389/fpsyg.2019.00619

Rubio-Garay, F., Carrasco, M. Á., Amor, P. J., \& López-González, M. A. (2015). Factores asociados a la violencia en el noviazgo entre adolescentes: una revisión crítica. Anuario de Psicología Jurídica, 25(1), 47-56. https://doi.org/10.1016/j. apj.2015.01.001

Rubio-Garay, F., López-González M. Á., Carrasco M.A. \& Amor P. J. (2017). Prevalencia de la violencia en el noviazgo: una revisión sistemática. Papeles del psicólogo, 38(2), 135-147. Recuperado de: https://dialnet.unirioja.es/servlet/articulo?codigo $=6029503$

Sociedad Mexicana de Psicología. (2007). Código ético del psicólogo (4ạ edición). México, D.F.: Editorial Trillas.

Straus, M. A. (1979). Measuring intrafamily conflict and violence: The Conflict Tactics (CT) Scales. Journal of Marriage and the Family, 41(1), 75-88. https://doi. org $/ 10.2307 / 351733$

Straus, M., \& Mickey, E. (2012). Reliability, validity, and prevalence of partner violence measured by the conflict tactics scales in male-dominant nations. Aggression and Violent Behavior, 17, 463-474. https://psycnet.apa.org/doi/10.1016/j. avb.2012.06.004

Valdivia, M. P., \& González, L. A. (2014). Violencia en el noviazgo y pololeo: una actualización proyectada hacia la adolescencia. Revista de Psicología, 32(2), 329355. Recuperado de: http://www.scielo.org.pe/scielo.php?pid=S0254-92472014 000200006\&script=sci_arttext

Vázquez-Sánchez, V., Romo-Tobón, R. J., Rojas-Solís, J. L., González Flores, M. D. P., $\&$ Rey Yedra, L. (2019). Violencia filio-parental en adultos emergentes mexicanos: Un análisis exploratorio. Revista Electrónica de Psicología Iztacala, 22(3), 25342551. Recuperado de: https://www.medigraphic.com/cgi-bin/new/resumen. cgi?IDARTICULO $=89678$

Wincentak, K., Connolly, J., \& Card, N. (2017). Teen dating violence: A meta-anaIytic review of prevalence rates. Psychology of Violence, 7(2), 224-241. https:// doi.org/10.1037/a0040194 